\title{
MINIREVIEW
}

\section{Acyldepsipeptide Antibiotics - Current State of Knowledge}

\author{
MICHAŁ T. PSTRĄGOWSKI ${ }^{1 \star}$ and MAGDALENA BUJALSKA-ZADROŻNY ${ }^{1}$ \\ ${ }^{1}$ Department of Pharmacodynamics, Medical University of Warsaw, CePT Laboratory, Warszawa, Poland
}

Submitted 12 June 2014, revised 19 January 2015, accepted 16 February 2015

\begin{abstract}
The objective of this paper is to review and summarize the antimicrobial efficacy of the acyldepsipeptides and to indicate the prospects of the therapeutic values of these compounds. This work is enriched by the description of the mutations within the $c l p P 1 c l p P 2$ and $c l p P 3 c l p P 4$ operons of Streptomyces lividans, which are considered to be the potential mechanism of the acyldepsipeptide (ADEP) - resistance development. The researchers' conclusions demonstrated a significant impact on microorganisms including the destabilization of bacterial cell division in Bacillus subtilis 168, Staphylococcus aureus HG001 and Streptococcus pneumoniae G9A strains. The results of animal studies show higher bactericidal effectiveness of the acyldepsipeptides ADEP-2 and ADEP-4 compared to linezolid. ADEPs may be considered as a very important mechanism of defense against the increasing resistance of microorganisms. They also might prevent or reduce the risk of many epidemiological events.
\end{abstract}

Ke y w ords: acyldepsipeptides, caseinolytic proteases A/X, drug resistance, Hsp100 proteins, microtubule, organization, proteins FtsZ

\section{Introduction}

The appearance of pathogens resistant to presently used antibiotics is now considered an increasingly serious problem, concerning not only healthcare facilities, but also patients treated for community-acquired infections. Among the main reasons for the growing epidemiological risk are: inappropriately conducted antibiotic treatments, different therapeutic standards in individual countries, the lack of standardized procedures controlling the effectiveness of treatment, overusage of antibiotics e.g. in veterinary and agriculture and increased population migrations worldwide. It is estimated that the proportion of nosocomial infections caused by methicillin-resistant Staphylococcus aureus (MRSA) strains increased in the United States' intensive care units from 2\% in 1974 to $64 \%$ in 2004 (Morell and Balkin, 2010). The last decade has seen the increased spread of vancomycin-resistant enterococci (Vancomycin-Resistant Enterococcus, VRE) and multidrug-resistant Streptococcus pneumoniae strains (especially PRSP - penicillin-resistant $S$. pneumoniae) considered to be one of the main causes of prolonged hospitalization and increased mortality of patients with respiratory tract infections (Hinzen et al., 2006).

The search for new molecules that exhibit different mechanisms of action than those characteristic for the presently used antibiotics are considered as the main defense line against the growing threat of microorganisms' increasing resistance. In this context, a new class of drugs with perspectives of antimicrobial efficacy may be acyldepsipeptides (ADEP), which interfere with the functioning of the protease complexes formed by $\mathrm{ClpA}$ and ClpP (ClpAP) and ClpX and ClpP (ClpXP), regulating the metabolism of proteins in prokaryotic cells.

It has been demonstrated that these compounds modify the enzyme's activity, switching it on uncontrolled proteolysis, resulting in degradation of key factors in terms of proper conduct of cell division. As a result, acyldepsipeptides are a group of compounds of potentially valuable therapeutic bactericidal properties.

\section{Structure and tasks of the caseinolytic proteases (Clp) family}

ClpA (Clp ATPase-activity subunit(s); subunit A of Clp, which possesses ATPase activities) and ClpX (Clp subunit X) belong to a diverse subfamily of AAA+ proteins (ATPases Associated with various cellular activities). These proteins use the energy derived from ATP for conformational changes and participate in numerous enzymatic processes in the cell, i.e. DNA replication, protein synthesis, degradation of biological

\footnotetext{
* Corresponding author: M. Pstrągowski, Department of Pharmacodynamics, Medical University of Warsaw, CePT Laboratory, Warszawa, Poland; e-mail: pstragowskim@gmail.com
} 
membranes, microtubule organization, signal transduction or gene expression regulation (Alexopoulos et al., 2012). ClpA oligomerizes as a single hexameric ring made out of the stacking of its two ATPase domains (Barreiro et al., 2011; Beuron et al., 1998; Truscott et al., 2011). The basic core of so arranged enzyme is the protein ClpP (Clp proteolytic subunit(s); subunit P of Clp, which has the proteolytic active site), of which each of 14 analogical mobile subunits is built from an aliphatic stem, spherical domain and the $\mathrm{N}$-terminal region. It is supposed that such construction of this complex provides the formation of a specific axial channel, responsible for the degradation of selected polypeptides in the interior of ClpP (Barreiro et al., 2011; Beuron et al., 1998; Ortega et al., 2000).

The above model assumes the existence of two types of interaction between the core of this complex and $\mathrm{ClpA}$ or ClpX. The first one is related to the presence of a highly conservative region of IGF/L, located on the surface of both discussed cellular proteases. The second one is based on the activity of N-terminal protein loop, located near the axial channel (Lowth et al., 2012; Lupas et al., 1997; Marsault and Peterson, 2011). It should be emphasized that the mobility and specific distribution of subunits and axial channels of the complex is regarded as a protective barrier, which protects complex proteins and peptides containing more than 5-10 amino acids from the access to the interior of ClpP (Szyk and Maurizi, 2006; Thompson et al., 1994; Truscott et al., 2011).

Genetic studies have established that $c l p P$ gene and genes encoding the AAA+ partners are essential for virulence of e.g., S. aureus (Frees et al., 2003; 2005), Listeria monocytogenes (Gaillot et al., 2000), S. pneumoniae (Kwon et al., 2003; 2004; Robertson et al., 2002) and for viability in e.g. Mycobacterium tuberculosis (Raju et al., 2012b; Roberts et al., 2013). According to biochemical studies, mycobacterial ClpP1 and ClpP2 form separate homoheptameric rings which gather into an active ClpP1P2 heterotetradecameric complex (Raju et al., 2012b; Akopian et al., 2012).

Drugs targeting ClpP are not presently in clinical use but they are worth further development.

\section{Enopeptines - acyldepsipeptides precursors}

Acyldepsipeptides antibiotics have the ability to connect to the core protein ClpP. The chemical structure of precursor molecules of this class, ADEP-1, is based on a lactone core arranged from five different amino acids with attached side chain $\left(\mathrm{C}_{7} \mathrm{H}_{9}\right)$, containing three unsaturated bonds (Alexopoulos et al., 2012; Szyk and Maurizi, 2006; Thompson et al., 1994). The unsaturated bond in $\alpha \beta$ position is considered to be crucial in biological activity, it takes the trans configuration (Hinzen et al., 2006). A similar chemical structure is typical for a group of enopeptine antibiotics, which first representatives A54556A and B, were isolated in 1982. Research showed that ADEP-1 belongs to A54556 complex (Brötz-Oesterhelt et al., 2005). It has been demonstrated that enopeptins, differing from ADEP-1 by side length of chain and the presence of acetylated phenylalanine molecule as well as analogue of serine substituted by nitro group, characterize by effectiveness with regard to certain Gram-negative and Grampositive bacteria (Koshino et al., 1991; Osada et al., 1991). The above-mentioned authors observed the antibacterial activity of enopeptin A, including MRSA strains, but there was no inhibition to fungi in tested concentrations (Osada et al., 1991).

\section{Acyldepsipeptide antibiotics classification}

It has been shown that the activation strength of the bacterial proteolytic system is also determined by the used acyldepsipeptide type. Brötz-Oesterhelt et al. (2005) suggest that the precursor of this class of molecules is ADEP-1, identified as one of the products of Streptococcus hawaiiensis NRRL 15010 microbial metabolism. In order to receive and identify the subsequent, closely structurally related acyldepsipeptide compounds, this strain was used for the specially prepared fermentation process to yield eight acyldepsipeptides closely structurally related. Then, six of them (marked ADEP-1 - ADEP-6) were qualified for further studies.

Evaluation of in vitro properties, and then animal studies have shown that the strength of the bactericidal activity of ADEP-2 and ADEP-4 far exceeds the effectiveness of ADEP-1 in relation to eradication of Gram-negative bacteria, including strains resistant to multiple antibiotics. It was further observed that the described higher activity was determined by prior elimination of the active agent from the cell or addition of compounds increasing the permeability of a biological membrane to the culture medium. Other acyldepsipeptide compounds obtained, did not exhibit the desired biological activity. Brötz-Oesterhelt et al. (2005) passed a cell lysate of B. subtilis through an ADEP-5 affinity column. Reported effect was only partial, because only one protein reminded specifically bound, which the abovementioned Authors identified as ClpP by $\mathrm{N}$-terminal sequencing. In turn, ADEP-3 (being R-epimer of ADEP-2), due to unfavorable conformational changes within the difluorofenyloalanine moiety shows no binding affinity with the enzyme subunit (Brötz-Oesterhelt et al., 2005).

It is also suggested that the activity of selected acyldepsipeptide compounds may exceed the activ- 
ity of other antibiotics showing different mechanisms of action. In in vivo studies in rodents infected with Enterococcus faecalis the effectiveness of ADEP-2 and ADEP-4 was significantly higher than the activity of linezolid - the compound which makes it impossible to connect to the $30 \mathrm{~S}$ and $50 \mathrm{~S}$ subunits of the bacterial ribosome, inhibiting the initial phase of protein synthesis (Colca et al., 2003). Moreover, the use of ADEP-4 $(12.5 \mathrm{mg} / \mathrm{kg} \mathrm{b.w.}$ ) for the treatment of sepsis caused by S. aureus contributed to a significant increase in survival of experimental animals and reduced the adverse effect of sepsis on parenchymal organs functioning (Brötz-Oesterhelt et al., 2005). Additionally, in comparison with linezolid ADEP-4 was also significantly more effective in S. pneumoniae infections (Colca et al., 2003). These reports seem to confirm another study conducted both in vitro and in animal models. Hinzen et al. (2006) noted that with MIC equal to $0.125 \mu \mathrm{g} / \mathrm{ml}$ of these antibiotics exhibit satisfactory efficacy against S. aureus strains. The high efficiency of this class of compounds has also been confirmed with regard to S. pneumoniae and E. faecalis.

Wolbachia is a Gram-negative bacteria causing inhibition of worm development. There is a limitation in treatment of its infections in children and pregnant/ breastfeeding women because antibiotics commonly used in treatment of this infection can be dangerous for abovementioned group of patients. Fighting Wolbachia is hard, because although it is a Gram-negative bacterium, it has a non-classical outer membrane. This fact was explored in studies of Schiefer et al. (2013), who examined influence of acyldepsipeptides on inhibition of bacterial growth. These authors tested ADEP- 1 and its synthetic derivatives - ADEP-2, ADEP-4, and ADEP-5 against Wolbachia. Comparing with doxycycline gold standard, they found that ADEP-1 removes Wolbachia as good as doxycycline. Less effective ADEP-5 achieved almost $80 \%$ reduction, while ADEP- 2 had $30 \%$ reduction and ADEP-4 had no significant effect on bacteria removal. Furthermore, tests comparing different dilutions of ADEP-1 and doxycycline showed that even at lowest concentration, ADEP- 1 was still effective allowing Wolbachia removal. In conclusion, the results obtained by Schiefer et al. (2013) showed that acyldepsipeptides dysregulated Wolbachia ClpP at very promising level.

\section{Acyldepsipeptide - structural modifications}

It is well-known that the conformational constraints of macrocylic molecules can be further enhanced by judicious introduction of substituents on the ring (Marsault and Peterson, 2011)

Undoubtedly acyldepsipeptides have antibacterial potential. Many scientists tried to modify their natural structures to obtain synthetic derivatives with higher efficacy. Socha et al. (2010) tested two strategies: one involving the replacement of the $\mathrm{N}$-methyl alanine moiety in the peptidolactone with $\alpha, \alpha$-disubstituted amino acids, and the second, involving the replacement of this residue with a substituted pipecolic acid. Scientists decided to optimize the structure of ADEP-4 as they thought it would be a better idea than synthesis of many new compounds. In further research, they have chosen compounds with more promising activity against pathogenic Staphylococci and Enterococci.

Carney et al. (2014a) in their studies related to the fact, that activities of acyldepsipeptides can be improved by replacing certain amino acid constituents in peptidolactone core with more conformationally constrained counterparts. In this study, noncovalent interactions between ADEPs and ClpP are used as the object of observations. Scientists made structural changes by replacing hydrogen to deuterium in peptides taking part in bounding ADEP-ClpP complex. The main hypothesis was confirmed by the data showing that the incorporation of conformationally constrained residues in the peptidolactone has a rigidifying effect on ADEP bounding. ATPases and ADEPs are competing for connecting to $\mathrm{ClpP}$ and obtained data showed that ADEP-ClpP bounding is tighter and stronger than ATPase binding. In conclusion, Carney et al. (2014a) reported that their modification of ADEP structure led to great improvements in the antimicrobial efficacy of these agents. The authors presented data that confirm replacement of selected amino acids constituents as being a good method to stabilize a bioactive conformation of ADEPs. Furthermore, in recent studies Carney et al. (2014b) identified the minimal structural component of the cyclic acyldepsipeptides that exhibits antibacterial activity. This active fragment is $\mathrm{N}$-acyldifluorophenylalanine and it operates via the same mechanism of action as ADEPs, it also needs ClpP for antibacterial activity (Carney et al., 2014b). Although having the same mode of action, $\mathrm{N}$-acylfluorophenylalanine fragments are much simpler in structure than the full ADEPs and are also highly amenable to structural diversification.

Structural changes of substituents on the ring are a well known method to enhance the conformational constraints among macrocylic molecules (Marsault and Peterson, 2011). These changes can be made by insertion of small methyl substituents profoundly enhancing the affinity of a large macrocycle for its biomolecular receptor as well as increasing biological activity of the molecule (Carney et al., 2014a). Replacement of hydrogen atoms with methyl groups is commonly used in structure-activity relationship (SAR) studies (Barreiro et al., 2011) but insertion of a methyl group into a ligand typically has deleterious effect or has no 
significant influence on receptor-binding properties of modified ligand (Carney et al., 2014a). This statement is confirmed by Leung et al. (2012) who demonstrated that in $8 \%$ of cases the inclusion of a methyl group enhances bioactivity tenfold and only in $0.4 \%$ of cases resulted in 100-fold enhancement of bioactivity. Leung et al. (2012) revealed also that the highest improvement is made by methyl group's capacity for filling a hydrophobic environment in the receptor, and by this influencing the conformation of the ligand.

The activity of acyldepsipeptides' also differs depending on the structural differences between their particles (Carney et al., 2014a). As it has already been stated by Hinzen et al. (2006), enopeptin A is more effective than enopeptin B against S.aureus, S.pneumoniae, E. faecalis and Enterococcus faecium. Difference between enopeptin A and B is that the first one has a 4-methylproline residue and the other have an unsubstituted proline residue (Hinzen et al., 2006). According to study by Carney et al. (2014a), scientists confirmed the importance of methyl substituent to acyldepsipeptide peptidolactone. Comparing unsubstituted molecule and either 4-methyl pipecolate or allo-threonine substituents in acyldepsipeptide particle. Carney et al. (2014a) found that allo-threonine have the strongest influence on conformational dynamics, ClpP affinity and bioactivity. Furthermore, inclusion of allo-threonine in acyldepsipeptide' peptidolactone improves MIC tenfold while 4-methylproline improves MIC only twofold (Hinzen et al., 2006). Despite this, both 4-methylproline and allo-threonine substituents lead to acquisition of acyldepsipeptides with lowest MICs ever reported for antibacterial agents (Carney et al., 2014a). ADEP-4 is reported as the most potent acyldepsipeptide able to cure S. aureus infections in mice and S. pneumoniae infections in rats with even greater efficacy than linezolid (Hinzen et al., 2006). Structural optimization led to obtaining analogs which have 200-fold lower MICs than ADEP-4 against S. pneumoniae and Escherichia coli. On this basis, we can speculate that potentially lower and safer doses of modified ADEPs analogues can be as much efficacious or even better than natural acyldepsipeptides (Carney et al., 2014a).

The next important issue is the cost of optimized compounds preparation. As it was presented by Hinzen et al. (2006), cost of insertion of allo-threonine residues into ADEP molecule is less and synthesis is easier than in case of 4-methylproline constituents of ADEP-4. Also, which is very important, observation made by scientists show that peptides with strong transannular hydrogen bonds have enhanced oral bioavailability. All these experiments lead to the conclusion that pharmacological properties of natural products can be improved by rational design of drug particle (Carney et al., 2014a).

\section{Effect of ADEP on Clp complex}

$\mathrm{X}$-ray studies have contributed to the development of two basic models of activating ClpP under the influence of ADEP. Biochemical studies indicate that ADEPs reprogram ClpP, converting it from a highly regulated peptidase that can degrade proteins only with the aid of its partner AAA + to an independent and unregulated protease (Lee et al., 2010). It is suggested that binding the antibiotic molecule results in disorder of $\mathrm{N}$-terminal structure of these fragment, which in physiological conditions are strongly stabilized by the surrounding hydrophobic groups (Lee et al., 2010).

ADEPs prevent formation of the complex between ClpP and ClpA ATPases and activate the independent $\mathrm{ClpP}$ core to degrade flexible proteins and nascent polypeptides in the course of translation (Lee et al., 2010). On the other hand, the alternative hypothesis says that the consequence of binding ADEP with ClpP is the formation of an open collar directly along the axis of symmetry of the complex core (Li et al., 2010). The assessment of the crystal ADEP-ClpP structure lead to conclusion that ADEPs influence ClpP and reprogram its physiological function. In analysis of Kirstein et al. (2006; 2009), ClpP of B. subtilis is a monomer which, in presence of ADEP, form higher oligomeric forms, while ClpP on its own is a monomer (Kirstein et al., 2006). For E. coli, the authors examined its ClpP and drawn a conclusion, that ADEP activates isolated $\mathrm{ClpP}$ in the absence of Clp-ATPase to degrade polypeptides but with lower processivity (Kirstein et al., 2009). Results from the studies of Brötz-Oesterhelt et al. (2005) show that purified native Bacillus $\mathrm{ClpP}$ is not able to degrade $\beta$-casein, a model substance for ClpP tests. Examination showed that immediately after addition of ADEP- 1 or ADEP-2 a complete casein degradation is triggered, and it occurs in absence of ATP-ases (Brötz-Oesterhelt et al., 2005). Furthermore, microscopic examination showed that after addition of ADEP-1 B. subtilis started to form long filaments instead of normal cells (Brötz-Oesterhelt et al., 2005). Based on above results we can say that mechanism of ADEP's action does not fall into one of the classical target areas, but involves direct or indirect inhibition of cell division (Brötz-Oesterhelt et al., 2005).

In the case of B. subtilis the conversion of ClpP into protease independent from ATP occurs, whereas in E. coli this process is conditioned mainly by stabilization of the whole complex, which allows the protein to penetrate to the metabolic center of the complex (Lee et al., 2010; Li et al., 2010; Dougan, 2011). Both models, however, indicate that the consequence of attaching the antibiotic is broadening the axial channel conditioned by conformational changes within the ClpP.

The studies conducted by Sowole et al. (2013), not only confirm previous assumptions, but also partly shed 
new light on the target points for ADEP actions. The study of proteolytic activity of the crystalline ClpP isolated from E. coli strains showed that ADEP anchors in the hydrophobic slots of the formed core of the complex, and then stabilizes the $\mathrm{N}$-terminal regions, so they take the conformation allowing broadening the axial channel and access to exposed bacterial proteins, including complex proteins and extended polypeptides that the described action may be dependent on the chemical structure. Li et al. (2010) suggest that ADEP long-chain alkyl residues $\left(\mathrm{C}_{7} \mathrm{H}_{9}\right)$, which function as a type of action sites stabilized by strong hydrophobic interactions, are responsible for keeping the active $\mathrm{ClpP}$ conformation (Lee et al., 2010; Li et al., 2010; Dougan, 2011).

Synthetic ADEP1 derivative, ADEP-4, has been tested for possible activation of ClpP. Conlon et al. (2013) presented that ADEP-4 has killing potential to bacteria. Scientists compared activity of different antibiotics and ADEP- 4 against $S$. aureus and found that in its ADEP-4 presence ClpP becomes a fairly nonspecific protease having abilities to stop bacterial growth. The fact, that null $c l p \mathrm{P}$ mutants are resistant to ADEP-4 (Brötz-Oesterhelt et al., 2005) was further explored by Conlon et al. (2013) and it was suggested that these mutants, despite the resistance to ADEP-4, are more susceptible to killing by many antibiotics. ADEP-4 resistant mutant seemed to be less tolerant to rifampicin and linezolid than the wild-type strain. In fact, when ADEP-4 was paired with rifampicin, this combination eradicated mutant $S$. aureus biofilm to the limit of bacteria detection (Conlon et al., 2013). Even tests with different strains of S. aureus (in the cited study strains: SA113, USA300, UAMS-1 and 37) confirmed big efficacy of ADEP-4-rifampicin combination to biofilm reduction.

Interesting observation was made by Lowth et al. (2012). Scientists generated a mutant human ClpX (hClpX) in which the mutation resulted in replacing glutamate residue within the Walker B motif of hClpX by alanine. Mutated protein still had the ability to bind ATP and, surprisingly, still was able to activate hClpP for degradation of model unfolded substrates like $\alpha$-casein (Lowth et al., 2012). Furthermore, the activation of hClpP was also possible in the presence of ADEP. For human ClpP, further experiments are required to determine if ADEP-like molecules can cause protein degradation in mitochondria, but also examination of possible mammalian hClpP-activators existence is necessary (Lowth et al., 2012).

\section{Effect of ADEPs influence on hsp100 proteins}

Hsp100 proteins (heat shock proteins with a molecular mass $100 \mathrm{kDa}$, subfamily of AAA+ proteins) are divided into two classes. Class 1 contains proteins with two AAA+ modules and includes such proteins as Hsp104, ClpB (Clp subunit B) and their distant relatives - ClpA and ClpC (Clp subunit C). Class 2 consists of proteins with one nucleotide-binding domain such as ClpX (Doyle and Wickner, 2009). ClpP creates a proteolytic complex with $\mathrm{Hsp} 100$ proteins which is mandatory for the degradation of polypeptides or proteins (Horwich et al., 1999; Sauer et al., 2004; Wickner et al., 1999). Studies conducted on isolated E. coli and B. subtilis, using fluorescein isothiocyanate-labeled casein confirmed that connecting ADEP-1 and ADEP-2 with ClpP-Hsp100 result in reprogramming of this complex (Horwich et al., 1999; Kirstein et al., 2006; 2008; 2009). Furthermore, ADEPs redirect the ClpP core to uncontrolled and deleterious degradation of unfolded substrates and can cause dissociation of ClpA/ClpP complex (Kirstein et al., 2009). Moreover, localization of ClpC and ClpX was the same in cells treated and untreated with ADEP which suggests that acyldepsipeptides have no influence on localization of Hsp100 proteins (Kirstein et al., 2008 ; 2009). In consequence, ADEP converts ClpP from a strongly regulated protease presenting high substrate specificity to an unrestrained and destructive proteolytic machinery (Kirstein et al., 2009).

\section{Effect of ADEP on organization of microtubules}

The direct consequence of acyldepsipeptide compounds' influence on complex of proteolytic enzymes can also be significant disruption of bacterial cells division. Sass et al. (2011) claimed that the use of small doses of ADEP affects the structure of bacteria, which was highlighted in studies conducted on B. subtilis 168, S. aureus HG001 and S. pneumoniae G9A strains. In the case of S. aureus HG001 and S. pneumoniae G9A bacterial cells swelled to nearly three times larger volume than the original ones. In turn, the B. subtilis 168 cells took the shape of long, regular filaments under the influence of ADEP molecules. The changes occurring in the tested microorganisms, allow to assume, that, despite the partial ability to biomass creation, they are completely devoid of regulatory features. The observations using electron microscopy confirmed association of septum formation inhibition and treating cells with ADEP, which makes cells unable to build a normal cell wall (Raju et al., 2012a; 2012b; Roberts et al., 2013; Robertson et al., 2002; Sass et al., 2011). Furthermore, Sass et al., (2011) investigated septum formation also in mutant of B. subtilis with a deletion of $c l p P$ gene in order to determine, whether the inhibition of septum formation was caused by ADEP-induced dysregulation of ClpP. At similar ADEP concentration Sass et al. (2011) observed no filamentation and normal cell division which showed crucial role of ClpP for ADEP activity. 


\section{Effect of ADEP on FtsZ proteins}

Among the factors sensitive to ADEP-ClpP complex proteolytic activity, superfamily of proteins FtsZ (Filamenting temperature-sensitive mutant $\mathrm{Z}$ ) is also mentioned. These structures, involved in formation of cytoskeleton prokaryotic cells, are homologous to tubuline and are able to hydrolyze GTP (Lan et al., 2009). It has been shown that the Fts $Z$ polymerization leads to the formation of ring, which initiates bacterial cell division into two progeny structures. One of the hypothesis assumes that this ring is a specific type of scaffold for other factors, which participate in the formation and maintenance of primary partition (Sass et al., 2011). However, another model suggests, that the complex formation may be related to the activity generated during the FtsZ polymerization of the peptide chains, which contribute to the creation of numerous tensions, compressing opposite cell edges (Kwon et al., 2003; 2004; Lan et al., 2009).

In vitro studies conducted by Alexopoulos et al. (2013), showed that destabilization of cell division leads to the formation of structures with unnatural appearance and modified properties. Additionally, it was confirmed that the exchange of proline to isoleucine within the $\mathrm{N}$ terminus of ClpP contributed to the complete loss of proteolytic activity against FtsZ. This confirms that ADEP's action may be closely related to a specific amino acid sequence of the complex. However, such dependencies were not observed in relation to proteins degraded by ADEP-ClpP. Sass et al. (2011) proved that, both bovine $\alpha \beta$-tubulin and FtsZ are metabolized in a similar way, and the only differences are associated with different length of peptide chains of final products of this process.

\section{Effect of acyldepsipeptide on Mycobacterium tuberculosis}

Acyldepsipeptide compounds were also tested for efficacy against $M$. tuberculosis. This bacteria has two homologs of ClpP protease, encoded by two different $\mathrm{ClpP}$ operons and marked successively as ClpP1 and ClpP2 (Ollinger et al., 2012). Both of these proteases, despite the high structural similarity, have different substrates, which may suggest that their activation entail different consequences for the vital functions of the cell. Studies performed by Compton et al. (2013) and Ollinger et al. (2012) revealed that overexpression of ClpP1 is well tolerated by M. tuberculosis strains in contrast to overexpression of $\mathrm{ClpP} 2$; the latter correlates with the appearance of toxic effects. However, on the grounds of fact that ClpP1 and ClpP2 are structural homologs. Ollinger et al. (2012) suggest that ADEPs would activate both mentioned ClpPs. These authors showed that acyldepsipeptides (ADEP-1, ADEP-2 and ADEP-3) and two synthetic structural antibiotics analogues of this class (IDR-10001 and IDR-10011) admittedly influence vital functions of M. tuberculosis, however, this effect is less distinct in comparison with the impact on more sensitive S. aureus. Differences in actions of studied compounds may be depending on increased amounts of lipid compounds in the structure of the mycobacterial cell wall, which also explains their specific mechanisms of resistance. Apart from that, the absence of a clpP3clpP4 locus in M. tuberculosis gives hope for use of acyldepsipeptides as antibacterial drug (Compton et al., 2013).

Ollinger et al. (2012) reported that although acyldepsipeptides are effective themselves, they must be used in combination with efflux-pump inhibitors to keep low MICs in case of M. tuberculosis. Results obtained in their study suggests that verapamil and reserpine are good efflux pump inhibitors for increasing the effectiveness of acyldepsipeptides. It is necessary to conduct a further studies for efflux pump inhibitors development, not only in terms of breaking the resistance of microorganisms but also for the implementation of a potential combination therapy (Ollinger et al., 2012).

\section{Potential mechanisms of resistance to ADEP}

The first reports on acyldepsipeptides suggest that full development of effective resistance mechanisms is characterized by low probability. Nonetheless, it should be remembered that similar assumptions (as things turned out later, groundless) were put forward when implementing the quinolone treatment. Even today, some authors consider the possibility that the process of developing resistance can occur on the pharmacogenomic level.

Gominet et al. (2011) showed that ClpP gene expression regulation is made through the clpP1clpP2 operon as well as single $c l p P 5$ gene. It is suggested that mutations within these operones may lead to biosynthesis of ClpP forms insensitive to acyldepsipeptides and, in consequence, to come into existence strains resistant to ADEPs. In order to verify the above hypothesis Gominet et al. (2011) evaluated the effectiveness of ADEP against mutated Streptomyces lividans strains where the mutation of $c l p P 1$ was made to confirm the resistance to acyldepsipeptides. It was revealed that clpP1 mutants show ClpP1 protease activity, however, it was much weaker in comparison with the wild type strains (Gominet et al., 2011). To confirm that the resistance of the $S$. lividans clpP1 mutant to ADEP was a consequence of the $c l p P 1$ mutation, Gominet et al. (2011) transformed the strain with $p V D C 742$ plasmid, 
carrying the wild-type $c l p P 1 c l p P$ operon. This multistage process led to full restoration of sensitivity to the acyldepsipeptide compounds (Gominet et al., 2011).

Furthermore, it was shown that the mutation within another operon, $\operatorname{clp} P 3 \operatorname{cl} p P 4$, is not identical with receiving ADEP-resistant strain. In practice, S. lividans strain, transformed with $p J V 41$ plasmid that leads to overexpression of $\operatorname{clpP} 3$ clpP4, is still sensitive to ADEP (Gominet et al., 2011). It is also assumed that ClpP3 overproduction itself, does not determine sensitivity to ADEP. In this case, the inverse relation is observed - ClpP3 is a factor insensitive to acyldepsipeptide compounds but $c l p P 3 c l p P 4$ mutants retain susceptibility to antibiotics of this class. Gominet et al. (2011) suggest also that they tried to overexpress clpP4 under control of ermE promoter and they found that there is no evidence for ADEP resistance in S. lividans mutant. Both ClpP4 and $\mathrm{ClpP} 5$ proteases have atypical, so-called, catalytic triad (conditioned by the presence of serine, histidine and aspartate) and probably have regulatory, not functional, role and therefore should not be taken into account when determining the detailed mechanisms of resistance to ADEP (Gominet et al., 2011).

Summary. Discovering new target points for antimicrobial compounds, along with full knowledge of resistance mechanisms, is considered one of the key points of progress in modern antibiotic therapy. The achievements of researchers described in this paper, indicate that the acyldepsipeptides belonging to the enopeptine class of antibiotics, could be a class of drugs giving perspectives of antimicrobial effectiveness. Activity associated with interference in cellular activity of proteolytic enzymes of Gram-positive bacteria, in vitro test results, as well as results of studies carried out in animal models seem to confirm that the new, unique mechanism of action of the described group of compounds based on the impact on ClpP complexes also determine their efficacy against strains resistant to the vast majority of presently used antibiotics (e.g. MRSA, M. tuberculosis, B. subtilis, S.pneumonia, E. faecalis). Additionally, this mechanism also prevents the rapid emergence of resistance, which, in the optimistic variant, can translate into a significant reduction of epidemiological risk in the coming years.

\section{Acknowledgements \\ This review received no specific grant from any funding agency in the public, commercial or not profit sectors.}

\section{Literature}

Alexopoulos J.A., A. Guarné and J. Ortega. 2012. ClpP: a structurally dynamic protease regulated by AAA+ proteins. J. Struct. Biol. 179: 202-210.
Alexopoulos J., B. Ahsan, L. Homchaudhuri, N. Husain, Y.Q. Cheng and J. Ortega. 2013. Structural determinants stabilizing the axial channel of ClpP for substrate translocation. Molecular Microbiology 90: $167-180$.

Akopian T., O. Kandror, R. Raju, M. UnniKrishnan, E. Rubin and A. Goldberg. 2012. The active ClpP protease from M. tuberculosis is a complex composed of a heptameric ClpP1 and a ClpP2 ring. EMBO J. 31: 1529-1541.

Barreiro E.J., A.E. Kümmerle and C.A. Fraga. 2011. The methylation effect in medicinal chemistry. Chem. Rev. 111: 5215-5246.

Beuron F., M.R. Maurizi, D.M. Belnap, E. Kocsis, F.P. Booy, M. Kessel and A.C. Steven. 1998. At sixes and sevens: characterization of the symmetry mismatch of the ClpAP chaperone-assisted protease. J. Struct. Biol. 123(3), 248-259.

Brötz-Oesterhelt H., D. Beyer, H.P. Kroll, R. Endermann, C. Ladel, W. Schroeder, B. Hinzen, S. Raddatz, H. Paulsen, K. Henninger and others. 2005. Dysregulation of bacterial proteolytic machinery by a new class of antibiotics. Nat. Med. 11: 1082-1087. Carney D.W., K.R. Schmitz, J.V. Truong, R.T. Sauer and J.K. Sello. 2014. Restriction of the conformational dynamics of the cyclic acyldepsipeptide antibiotics improves their antibacterial activity. J. Am. Chem. Soc. 136: 1922-1929.

Carney D.W., C.L. Compton, K.R. Schmitz, J.P. Stevens, R.T. Sauer and J.K. Sello. 2014. A simple fragment of cyclic acyldepsipeptides is necessary and sufficient for ClpP activation and antibacterial activity. Chembiochem. 10.1002/cbic.201402358.

Colca J.R., W.G. McDonald, D.J. Waldon, L.M. Thomasco, R.C. Gadwood, E.T. Lund, G.S. Cavey, W.R. Mathews, L.D. Adams, Cecil E.T. and others. 2003. Cross linking in the living cell locates the site of action of oxazolidinone antibiotics. J. Biol. Chem. 278: 21972-21979.

Compton C.L., K.R. Schmitz, R.T. Sauer and J.K. Sello. 2013. Antibacterial activity of and resistance to small molecule inhibitors of the ClpP peptidase. ACS Chem. Biol. 8: 2669-2677.

Conlon B.P., E.S. Nakayasu, L.E. Fleck, M.D. LaFleur, V.M. Isabella, K. Coleman, S.N. Leonard, R.D. Smith, J.N. Adkins and K. Lewis. 2013. Activated ClpP kills persisters and eradicates a chronic biofilm infection. Nature 503: 365-70.

Dougan D.A., B.G. Reid, A.L. Horwich and B. Bukau. 2002. ClpS, a substrate modulator of the ClpAP machine. Mol. Cell. 9: 673-683. Dougan D.A. 2011. Chemical activators of ClpP: turning Jekyll into Hyde. Chem. Biol. 18: 1072-1074.

Doyle S.M. and S. Wickner. 2009. Hsp104 and ClpB: protein disaggregating machines. Trends Biochem. Sci. 34: 40-48.

Frees D., S.N.A. Qazi, P.J. Hill and H. Ingmer. 2003. Alternative roles of ClpX and ClpP in Staphylococcus aureus stress tolerance and virulence. Mol. Microbiol. 48: 1565-1578.

Frees D., K. Sorensen and H. Ingmer. 2005. Global virulence regulation in Staphylococcus aureus: pinpointing the roles of ClpP and ClpX in the sar/agr regulatory network. Infect. Immun. 73: 8100-8108.

Gaillot O., E. Pellegrini, S. Bregenholt, S. Nair and P. Berche. 2000. The ClpP serine protease is essential for the intracellular parasitism and virulence of Listeria monocytogenes. Mol. Microbiol. 35: 1286-1294.

Gominet M., N. Seghezzi and P. Mazodier. 2011. Acyl depsipeptide (ADEP) resistance in Streptomyces. Microbiology. 157: 2226-2234. Hinzen B., S. Raddatz, H. Paulsen, T. Lampe, A. Schumacher, D. Häbich, V. Hellwig, J. Benet-Buchholz, R. Endermann, H. Labischinski and others. 2006. Medicinal chemistry optimization of acyldepsipeptides of the enopeptin class antibiotics. Chem. Med. Chem. 1: 689-693.

Horwich A.L., E.U. Weber-Ban and D. Finley. 1999. Chaperone rings in protein folding and degradation. Proc. Natl. Acad. Sci. USA 96: $11033-11040$ 
Kirstein J., T. Schlothauer, D.A. Dougan, H. Lilie, G. Tischendorf, A. Mogk, B. Bukau and K. Turgay. 2006. Adaptor protein controlled oligomerization activates the $\mathrm{AAA}+$ protein $\mathrm{ClpC}$. EMBO J. 25: 1481-1491.

Kirstein J., H. Strahl, N. Moliere, L.W. Hamoen and K. Turgay. 2008. Localization of general and regulatory proteolysis in Bacillus subtilis cells. Mol. Microbiol. 70: 682-694.

Kirstein J., A. Hoffmann, H. Lilie, R. Schmidt, H. RübsamenWaigmann, H. Brötz-Oesterhelt, A. Mogk and K. Turgay. 2009. The antibiotic ADEP reprogrammes ClpP, switching it from a regulated to an uncontrolled protease. EMBO Mol. Med. 1: 37-49.

Koshino H., H. Osada, T. Yano, J. Uzawa and K. Isono. 1991. The structure of enopeptins A and B, novel depsipeptide antibiotics. Tetrahedron Lett. 32: 7707-7710.

Kwon H., S. Kim, M. Choi, A.D. Ogunniyi, J.C. Paton, S. Park, S. Pyo and D. Rhee. 2003. Effect of heat shock and mutations in $\mathrm{ClpL}$ and $\mathrm{ClpP}$ on virulence gene expression in Streptococcus pneumoniae. Infect. Immun. 71: 3757-3765.

Kwon H., A.D. Ogunniyi, M. Choi, S. Pyo, D. Rhee and J.C. Paton. 2004. The ClpP protease of Streptococcus pneumoniae modulates virulence gene expression and protects against fatal pneumococcal challenge. Infect. Immun. 72: 5646-5653.

Lan G., B.R. Daniels, T.M. Dobrowsky, D. Wirtz and S.X. Sun. 2009. Condensation of FtsZ filaments can drive bacterial cell division. Proc. Natl. Acad. Sci. U.S.A. 106: 121-126.

Lee B.G., E.Y. Park, K.E. Lee, H. Jeon, K.H. Sung, H. Paulsen, H. Rübsamen-Schaeff, H. Brötz-Oesterhelt and H.K. Song. 2010. Structures of ClpP in complex with acyldepsipeptide antibiotics reveal its activation mechanism. Nat. Struct. Mol. Biol. 17: 471-478.

Leung C.S., S.S. Leung, J. Tirado-Rives and W.L. Jorgensen. 2012. Methyl effects on protein-ligand binding. J. Med. Chem. 55: 4489-4500.

Li D.H., Y.S. Chung, M. Gloyd, E. Joseph, R. Ghirlando, G.D. Wright, Y.Q. Cheng, M.R. Maurizi, A. Guarné and J. Ortega. 2010. Acyldepsipeptide antibiotics induce the formation of a structured axial channel in ClpP: A model for the ClpX/ClpA-bound state of ClpP. Chem. Biol. 17: 959-969.

Lowth B.R., J. Kirsten-Miles, T. Saiyed, H. Brötz-Oesterhelt, R.I. Morimoto, K.N. Truscott and D.A. Dougan. 2012. Substrate recognition and processing by a Walker B mutant of the human mitochondrial AAA+ protein CLPX. J. Struct. Biol. 179: 193-201.

Lupas A., J.M. Flanagan, T. Tamura and W. Baumeister. 1997. Selfcompartmentalizing proteases. Trends Biochem. Sci. 22: 399-404.

Marsault E. and M.L. Peterson. 2011. Macrocycles are great cycles: applications, opportunities, and challenges of synthetic macrocycles in drug discovery. J. Med. Chem. 54: 1961-2004.

Morell E.A. and D.M. Balkin. 2010. Methicillin-resistant Staphylococcus aureus: a pervasive pathogen highlights the need for new antimicrobial development. Yale J. Biol. Med. 83: 223-233.

Ollinger J., T. O'Malley, E.A. Kesicki, J. Odingo and T. Parish. 2012. Validation of the essential ClpP protease in Mycobacterium tuberculosis as a novel drug target. J. Bacteriol. 194: 663-668.
Ortega J., S.K. Singh, T. Ishikawa, M.R. Maurizi and A.C. Steven. 2000. Visualization of substrate binding and translocation by the ATP-dependent protease, ClpXP. Mol. Cell. 6: 1515-1521.

Osada H., T. Yano, H. Koshino and K. Isono. 1991. Enopeptin A, a novel depsipeptide antibiotic with anti-bacteriophage activity. J. Antibiot. 44: 1463-1466.

Raju R.M., A.L. Goldberg and E.J. Rubin. 2012. Bacterial proteolytic complexes as therapeutic targets. Nat. Rev. Drug Discov. 11: 777-789.

Raju R., M. Ennikrishnan, D. Rubin, V. Krishnamoorthy, O. Kandror, T. Akopian, A. Goldberg and E. Rubin. 2012. Mycobacterium tuberculosis $\mathrm{ClpP} 1$ and $\mathrm{ClpP} 2$ function together in protein degradation and are required for viability in vitro and during infection. PLoS Pathog. 8: e1002511.

Roberts D.M., Y. Personne, J. Ollinger and T. Parish. 2013. Proteases in Mycobacterium tuberculosis pathogenesis: potential as drug targets. Future Microbiol. 8: 621-631.

Robertson G.T., W. Ng, J. Foley, R. Gilmour and M.E. Winkler. 2002. Global transcriptional analysis of clpP mutations of type 2 Streptococcus pneumoniae and their effects on physiology and virulence. J. Bacteriol. 184: 3508-3520.

Sass P., M. Josten, K. Famulla, G. Schiffer, H.G. Sahl, L. Hamoen and H. Brötz-Oesterhelt. 2011. Antibiotic acyldepsipeptides activate ClpP peptidase to degrade the cell division protein FtsZ. Proc. Natl. Acad. Sci. USA 108: 17474-17479.

Sauer R.T., D.N. Bolon, B.M. Burton, R.E. Burton, J.M. Flynn, R.A. Grant, G.L. Hersch, S.A. Joshi, J.A. Kenniston, I. Levchenko and others. 2004. Sculpting the proteome with AAA+. proteases and disassembly machines. Cell. 119: 9-18

Schiefer A., J. Vollmer, C. Lämmer, S. Specht, C. Lentz, H. Ruebsamen-Schaeff, H. Brötz-Oesterhelt, A. Hoerauf and K. Pfarr. 2013. The ClpP peptidase of Wolbachia endobacteria is a novel target for drug development against filarial infections. J. Antimicrob. Chemother. 68: 1790-1800.

Socha A.M., N.Y. Tan, K.L. LaPlante, J.K. Sello. 2010. Diversityoriented synthesis of cyclic acyldepsipeptides leads to the discovery of a potent antibacterial agent. Bioorg. Med. Chem. 18: 7193-7202 Sowole M.A., J.A. Alexopoulos, Y.Q. Cheng, J. Ortega and L. Konermann. 2013. Activation of ClpP protease by ADEP antibiotics: insights from hydrogen exchange mass spectrometry. J. Mol. Biol. 425: 4508-4519.

Szyk A. and M.R. Maurizi. 2006. Crystal structure at 1.9Å of E. coli $\mathrm{ClpP}$ with a peptide covalently bound at the active site. J. Struct. Biol. 156: 165-174.

Thompson M.W., S.K. Singh and M.R. Maurizi. 1994. Processive degradation of proteins by the ATP-dependent Clp protease from Escherichia coli. Requirement for the multiple array of active sites in ClpP but not ATP hydrolysis. J. Biol. Chem. 269: 18209-18215.

Truscott K.N., A. Bezawork-Geleta and D.A. Dougan. 2011. Unfolded protein responses in bacteria and mitochondria: a central role for the ClpXP machine. IUBMB Life. 63: 955-963

Wickner S., M.R. Maurizi and S. Gottesman. 1999. Posttranslational quality control: folding, refolding, and degrading proteins. Science 286: 1888-1893 